\title{
Compatibilidad y tiempo de sobrevivencia de cuatro microorganismos benéficos de uso agrícola en biol
}

\section{Compatibility and survivorship of four beneficial microorganism used in boils in agriculture}

\author{
Villacís-Aldaz Luis ${ }^{*}$, Chungata Luis, Pomboza Pablo, León Olguer
}

\begin{tabular}{l}
\hline Datos del Articulo \\
\hline Facultad de Ciencias Agropecuarias. \\
Universidad Técnica de Ambato. \\
Tungurahua - Ecuador. \\
Casilla postal: 18-01-334. \\
Telf: (+593)032872630-0985471191 \\
luis.chungata@ hotmail.com \\
ptamaquiza@ @mail.com \\
olguerleon@yahoo.es \\
*Dirección de contacto: \\
Luis Villacís-Aldaz \\
Cantón Cevallos-Tungurahua. Ecuador. \\
Casilla postal: 18-01-334. \\
Telf: (+593) 032872630-0985471191. \\
E-mail: la.villacis@uta.edu.ec \\
\hline Palabras clave: \\
\hline Agroecológico, \\
biol, \\
hongos, \\
microbiológico, \\
pH, \\
sustentable.
\end{tabular}

\section{Resumen}

Se evaluó la compatibilidad y sobrevivencia de microorganismos benéficos presentes en bioles de uso agrícola con el propósito de generar nuevas tecnologías de producción sustentables. El estudio fue realizado en una granja agroecológica del Gobierno Provincial de Tungurahua. Se evaluó la sobrevivencia y compatibilidad de Trichoderma harzianum, Metarhizium anisopliae, Beauveria bassiana y Paecilomyces lilacinus en un biol de elaboración artesanal. Los tratamientos consistieron en aplicar $0 \mathrm{~mL}$ (T0), $50 \mathrm{~mL}$ (T1), $100 \mathrm{~mL}$ (T2) y $150 \mathrm{~mL}$ (T3) de la combinación de los microrganismos en $20 \mathrm{~L}$ de biol. A los 30 días, la mayor población de Metarrhizium, Trichoderma y Beauveria fue observado en el T3 $\left(9.1 \times 10^{5} \mathrm{UPC} / \mathrm{mL}\right.$ de biol) con pH 3.80. A los 60 días, se verificó que la mayor población fue observada en el T2 $\left(2,1 \times 10^{6} \mathrm{UPC} / \mathrm{mL}\right)$ a $\mathrm{pH} 4.95$. Se observó que los géneros Paecilomyces y Beauveria no crecieron juntos, lo que permite sugerir una posible incompatibilidad.

(C) 2016. Journal of the Selva Andina Biosphere. Bolivia. Todos los derechos reservados.

J Selva Andina Biosph. 2016; 4(1):39-45.

\section{Historial del artículo.}

Recibido febrero, 2016

Devuelto abril 2016

Aceptado mayo, 2016.

Disponible en línea, mayo 2016

\section{Editado por:}

Selva Andina Re-

search Society

\section{Key words:}

Agro ecological,

Boil,

Fungi,

Microbiological,

$\mathrm{pH}$,

sustainable.

\section{Abstract}

Compatibility and survival of a mixture of beneficial microorganisms in a biol used in agriculture were evaluated in order to generate new sustainable production technologies. The study was carried out in an agro ecological farm of the Provincial Government of Tungurahua. Survival and compatibility of Trichoderma harzianum, Metarhizium anisopliae, Beauveria bassiana and Paecilomyces lilacinus in a homemade boil were evaluated. Treatments consisted in adding 0 $\mathrm{mL}$ (T0), $50 \mathrm{~mL}$ (T1), $100 \mathrm{~mL}$ (T2) or $150 \mathrm{~mL}$ (T3) of a microorganism combination in $20 \mathrm{~L}$ biol. At day 30, higher population of Metarrhizium, Trichoderma and Beauveria was observed in T3 $\left(9.1 \times 10^{5} \mathrm{UPC} / \mathrm{mL}\right.$ biol $)$ at $\mathrm{pH} 3.80$. At day 60 , higher population was verified in $\mathrm{T} 2\left(2,1 \times 10^{6} \mathrm{UPC} / \mathrm{mL}\right)$ at $\mathrm{pH} 4.95$. Genera Paecilomyces and Beauveria were not observed growing together, suggesting a possible incompatibility between them. 


\section{Introducción}

La continua demanda de aumento en la producción y rendimiento de cultivos ha provocado el incremento en el uso de fertilizantes químicos en la agricultura (Pino 2009). Adicionalmente, la creciente susceptibilidad de algunos cultivos al ataque de plagas también ha favorecido el uso de plaguicidas en detrimento de las poblaciones de microorganismos benéficos del suelo (Sánchez 2009). Este hecho ha ocasionado problemas de contaminación ambiental por la acumulación de tóxicos, entre los cuales se incluyen los metales pesados cuya incorporación a las cadenas alimentarias ha acarreado problemas de salud humana (Semu \& Singh 1996). Por otra parte, la microflora y fauna del suelo son de ponderable importancia ecológica, sin embargo, actualmente el equilibrio ecológico de estos organismos ha sido alterado por el uso excesivo de agroquímicos (Campo-Martínez et al. 2014).

La contaminación de los alimentos debido al uso excesivo de fungicidas sintéticos constituye un problema concomitante, por lo que la utilización de microorganismos benéficos para sustituir el uso de agroquímicos representa una alternativa a escala mundial (Ferrera-Cerrato \& Alarcón 2001, Navia et al. 2013). La combinación de varios microorganismos benéficos (EMs) fue desarrollada en la Universidad de Ryukyus en Okinawa, Japón en 1980, quienes comenzaron la búsqueda de una alternativa para la producción de alimentos en el mundo entero. Inicialmente los EMs se utilizaron como acondicionador del suelo y actualmente son también usados para producir alimentos de altísima calidad, libres de agroquímicos, así como para el manejo de desechos sólidos y líquidos generados por la producción agropecuaria, la industria de procesamiento de ali mentos, fábricas de papel, mataderos y municipalidades, entre otros (Zapata 2015).

La presente investigación fue realizada con el fin de evaluar la compatibilidad y el tiempo de sobrevivencia de cuatro microorganismos benéficos (Trichoderma harzianum, Metarhizium anisopliae, Beauveria bassiana y Paecilomyces lilacinus) de uso agrícola en los bioles, con el propósito de obtener cultivos limpios y garantizando técnicas amigables con el medio ambiente.

\section{Materiales y métodos}

El estudio se desarrolló en la Granja Agroecológica Píllaro perteneciente al Consejo Provincial de Tungurahua, Ecuador $\left(01^{\circ} 10^{\prime} \mathrm{S}\right.$; 78 $\left.32^{\circ} \mathrm{W}\right)$, ubicada a $2.825 \mathrm{msnm}$, con temperatura promedio de $13.5^{\circ} \mathrm{C}$ (Datos obtenidos con GPS Garmin).

\section{Tabla 1 Insumos usados para preparar $200 \mathrm{~L}$ de biol}

\begin{tabular}{lccc}
\hline Insumos & Cantidad & Unidad & Presentación \\
\hline Estiércol fresco de bovino & 60 & $\mathrm{lb}$ & Semi-sólido \\
Hierbas frescas aromáticas & 5 & $\mathrm{lb}$ & Sólido \\
Hierbas frescas leguminosas & 10 & $\mathrm{lb}$ & Sólido \\
Sulfato de cobre & 2 & $\mathrm{lb}$ & Sólido \\
Carbonato de calcio & 2 & $\mathrm{lb}$ & Sólido \\
Levadura de cerveza & 2 & $\mathrm{lb}$ & Sólido \\
Melaza & 30 & 1 & Líquido \\
Roca fosfórica & 4 & $\mathrm{lb}$ & Sólido \\
Sulfato de potasio & 4 & $\mathrm{lb}$ & Sólido \\
Bórax & 200 & $\mathrm{~g}$ & Sólido \\
Azufre micronizado & 200 & $\mathrm{~g}$ & Sólido \\
Sulfato de hierro & 100 & $\mathrm{~g}$ & Sólido \\
Sulfato de magnesio & 200 & $\mathrm{~g}$ & Sólido \\
\hline
\end{tabular}

Se evaluó la sobrevivencia y compatibilidad de diferentes microorganismos benéficos en un biol de 
elaboración artesanal (Tabla 1). Los microorganismos evaluados fueron Trichoderma harzianum, $\left(2.5 \times 10^{9} \mathrm{UPC} / \mathrm{g}\right.$ de sustrato), Metarhizium anisopliae, $\left(2.5 \times 10^{9} \mathrm{UPC} / \mathrm{mL}\right.$ de producto), Beauveria bassiana $\left(2.5 \times 10^{9} \mathrm{UPC} / \mathrm{mL}\right.$ de producto) y Paecilomyces lilacinus $\left(2.5 \times 10^{9} \mathrm{UPC} / \mathrm{mL}\right.$ producto $)$, adquiridos en la Escuela Superior Politécnica de Chimborazo, los cuales fueron adicionados al biol y mantenidos bajo cubierta en recipientes de $200 \mathrm{~L}$. Los tratamientos consistieron en aplicar 0 (T0), 50 (T1), 100 (T2) y $150 \mathrm{~mL}$ (T3) de la combinación de los microrganismos en $20 \mathrm{~L}$ de biol.

Las variables evaluadas fueron: número poblacional y compatibilidad de los EMs. Adicionalmente se midió $\mathrm{pH}$, conductividad eléctrica y densidad del biol. En cada tratamiento se tomaron muestras de $100 \mathrm{~mL}$ a los 30 y 60 días después de iniciada la inoculación. Cada muestra fue colocada en frascos de vidrio herméticamente cerrados, rotulados y enviados al laboratorio de fitopatología de la Escuela Superior Politécnica de Chimborazo para su identificación mediante el Método de Placa Pobre o Extensión.

El ensayo fue conducido en un diseño de completamente al azar con tres repeticiones. Los resultados fueron sometidos a análisis de varianza y las variables que mostraron diferencias significativas fueron sometidas a prueba de medias según Tukey $(\mathrm{p}<0.05)$ usando el paquete estadístico Infostat, versión estudiantil.

\section{Resultados}

En el análisis microbiológico antes de la inoculación de EMs mostró la presencia de Penicillium sp., Aspergillius sp., Alternaria sp. y Rhyzopus sp. (Tabla 2).

41
Tabla 2 Análisis microbiológico del biol antes de la inoculación de los EMs.

\begin{tabular}{lc}
\hline Hongos & Cantidad UPC/mL \\
\hline \hline Aspergillius $\mathrm{sp}$. & $3.0 \times 10^{4}$ \\
Rhizopus $\mathrm{sp}$. & $2.0 \times 10^{4}$ \\
Alternaria $\mathrm{sp}$. & $2.0 \times 10^{4}$ \\
Penicillium $\mathrm{sp}$. & $1.0 \times 10^{5}$ \\
\hline
\end{tabular}

UPC/mL: Unidad propagadora de colonias

Número de unidades propagadoras de colonias $(U P C)$. Se observaron diferencias significativas en el número promedio de UPC/mL a los 30 y 60 días después del inicio del ensayo $\left(\mathrm{p}_{30 \text { dias }}<0.0148, \mathrm{~F}\right.$ : 6.60, gl: 11 y $\mathrm{p}_{60 \text { dias }}<0.000$, F: 1243.72, gl: 11) (Fig. $1)$.

Figura 1 Número promedio de Unidades Propagadoras de colonias (UPC/mL) a los 30 y 60 días de inoculado

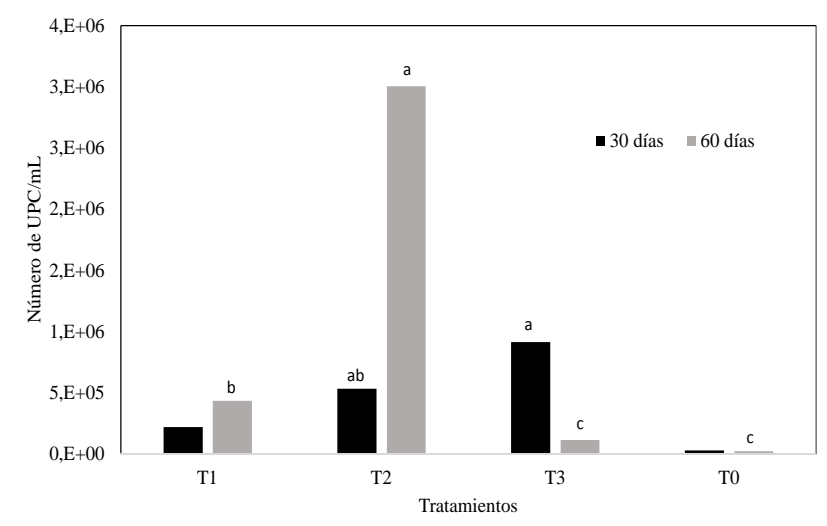

A los 30 días, se observó que el número promedio de UPC/mL fue de $2.2 \times 10^{5}$ en el biol tratado con 50 $\mathrm{mL}$ de microorganismos, mientras que se observó un incremento de 2.45 y 4.20 veces en el biol donde se adicionaron 100 y $150 \mathrm{~mL}$ de microorganismos, respectivamente. El mayor número de UPC/mL (2.1 $\left.\mathrm{x} 10^{6}\right)$ se lo encontró en el tratamiento T2 $(100 \mathrm{~mL}$ de cada uno de los EMs), seguido del T3 (150 mL de cada uno de los EMs), con un pH de 4.72 con un 
numero de UPC/mL, 8.6 × $10^{5}$ ubicándose en último lugar al testigo con el menor promedio de $1.6 \times 10^{4}$ $\mathrm{UPC} / \mathrm{mL}$.

En la evaluación hecha 60 días después de la inoculación también se observó un incremento en el número de UPC/mL en los diferentes tratamientos, sin embargo este número fue significativamente mayor en el biol donde se agregaron $100 \mathrm{~mL}$ de microorganismos. Por otra parte, la inoculación de los EMs provocó un incremento de nutrientes en nitrógeno, fósforo, calcio y magnesio (Tabla 3).
En el análisis de compatibilidad y el tiempo de sobrevivencia de los microorganismos a los 30 días se observaron niveles poblacionales altos de los géneros de hongos Trichoderma sp., Metarhizium sp. y Beauveria sp., con $9.1 \times 10^{5} \mathrm{UPC} / \mathrm{mL}$, en el T3, el cual tenía un $\mathrm{pH}$ 3.80. En el segundo análisis (60 días), se encontraron mayores niveles poblacionales de los hongos Trichoderma sp., Metarhizium sp., y Paecilomyces sp., con relación a los 30 días, siendo de $2.1 \times 10^{6} \mathrm{UPC} / \mathrm{mL}$ en el T2.

Tabla 3 Análisis químico del biol antes y después de la inoculación de los EMs.

\begin{tabular}{lllllllll}
\hline 0 a 60 días & $\mathbf{p H}$ & $\begin{array}{l}\mathbf{C E} \\
\mathbf{c s} / \mathbf{c m}\end{array}$ & $\begin{array}{l}\mathbf{N} \\
\mathbf{\%}\end{array}$ & $\begin{array}{l}\mathbf{P} \\
\mathbf{\%}\end{array}$ & $\begin{array}{l}\mathbf{K} \\
\mathbf{\%}\end{array}$ & $\begin{array}{l}\mathbf{C a} \\
\mathbf{\%}\end{array}$ & $\begin{array}{l}\mathbf{M g} \\
\mathbf{\%}\end{array}$ & $\begin{array}{l}\mathbf{F e} \\
\mathbf{p p m}\end{array}$ \\
\hline Antes & 5,95 & 21,12 & 0,21 & 0,35 & 0.87 & 0,96 & 0,16 & 1,38 \\
Después & 5,0 & 28,16 & 1,50 & 2,21 & 0,38 & 2,40 & 0,33 & 0,005 \\
\hline
\end{tabular}

Características físicas del biol. Los resultados de las variables físico-químicas del biol (Tabla 4). En general, se observó un incremento en los valores de $\mathrm{pH}, \mathrm{CE}$ y la densidad del biol a los 60 días con relación a los valores observados a los 30 días. Los va- lores de $\mathrm{pH}$ estuvieron en el rango de ligeramente ácido, con valores de 4.05, 3.73, 4.05 y 3.80 para los tratamientos T0, T1, T2 y T3, respectivamente, los cuales se incrementaron en $7.4,44.8,16.5$ y $30.3 \%$, respectivamente.

Tabla 4 Propiedades físico-químicas del biol a 30 y 60 días después de inoculados con los EMs

\begin{tabular}{|c|c|c|c|c|c|c|}
\hline \multirow[t]{2}{*}{ Inóculo } & \multicolumn{2}{|c|}{$\mathrm{pH}$} & \multicolumn{2}{|c|}{ CE mmhos $/ \mathrm{cm}$} & \multicolumn{2}{|c|}{ Densidad } \\
\hline & 30 días & 60 días & 30 días & 60 días & 30 días & 60 días \\
\hline 0 & $4.05 \pm 0.000$ & $4.35 \pm 0.000$ & $20.37 \pm 0.252$ & $27.07 \pm 0.252$ & $1.00 \pm 0.000$ & $1.01 \pm 0.000$ \\
\hline $50 \mathrm{ml}$ & $3.73 \pm 0.161$ & $5.40 \pm 0.400$ & $20.30 \pm 0.000$ & $28.40 \pm 0.265$ & $0.98 \pm 0.016$ & $1.01 \pm 0.005$ \\
\hline $100 \mathrm{ml}$ & $4.05 \pm 0.134$ & $4.72 \pm 0.765$ & $18.22 \pm 10.519$ & $29.87 \pm 0.058$ & $0.99 \pm 0.005$ & $1.01 \pm 0.001$ \\
\hline $150 \mathrm{ml}$ & $3.80 \pm 0.180$ & $4.95 \pm 0.695$ & $23.05 \pm 0.050$ & $25.60 \pm 0.100$ & $0.99 \pm 0.574$ & $1.00 \pm 0.002$ \\
\hline
\end{tabular}

Con relación a la CE, ésta mostró un incremento que varió entre 32.9 y $63.9 \%$ en los tratamientos control y T2 (100 mL de microorganismos), mientras que en el tratamiento donde fueron adicionados $150 \mathrm{~mL}$ de microorganismos la variación a los
60 días fue de apenas $11.1 \%$ con respecto al valor a los 30 días. 


\section{Discusión}

La presencia de Penicillium sp., Aspergillius sp., Alternaria sp., y Rhyzopus sp., pudo ser debido al tipo de insumos utilizados en la preparación del biol. Estos microorganismos han mostrado alta capacidad para producir antibióticos y micotoxinas que compiten con otras especies fúngicas (Arias et al. 2008). Por otro lado, este grupo de hongos posee un complejo de enzimas que les permite aprovechar el fósforo y el nitrógeno o degradar ácidos nucleicos y glicerofosfatos o fosfatos simples. De acuerdo con Díaz et al. (2009), estos hongos son saprófitos, habitantes naturales de materiales en descomposición por lo que es razonable encontrarlos en niveles poblacionales altos.

En los resultados del laboratorio con microorganismos en los primeros 30 días, tanto Penicillium y Aspergillius fueron encontrados en bajas poblaciones, demostrando la ubicuidad y la capacidad de crecer en diferentes temperaturas sobre sustratos o un caldo de cultivo, producen una amplia gama de antibióticos y micotoxinas, dificultando el crecimiento de otras especies. Los géneros de hongos Trichoderma sp., Metharhizum sp., y Beauveria sp., fueron encontrados en poblaciones altas sugiriendo que existe compatibilidad entre ellos.

Contrario a lo esperado, se obtuvo un mayor número de UPC/mL en el biol tratado con $100 \mathrm{~mL}$ de inóculo. Aunque el tamaño del inóculo tiene una influencia importante sobre el crecimiento de un microorganismo (Velastegui 2005, Bidlas et al. 2008), otros factores tales como $\mathrm{pH}$, temperatura y presión de vapor parcial también pueden favorecer o no el crecimiento de los microorganismos (Leistner 2000). Basados en los estudios de Velas43 tegui (2005), el pH podría ser considerado el factor determinante en el crecimiento microbiano, sugiriendo que estos microorganismos benéficos pueden reproducirse entre valores de $\mathrm{pH}$ entre 4.0 y 6.5. En tal sentido, probablemente el valor de $\mathrm{pH}$ alcanzado en el $\mathrm{T} 2(\mathrm{pH}=4.05)$ provocó un mayor incremento del número de UPC/mL al compararlo con el T3, donde dado el mayor inóculo, se esperaba que tuviera mayor incremento. Adicionalmente, la cantidad inicial de inóculo pudo haber influenciado favorablemente la multiplicación de los EMs y el control biológico de agentes patógenos, tal como fue evidenciado por el mayor número de $\mathrm{UPC} / \mathrm{mL}$ de $T$. harzianum, $P$. lilacinus, $M$. anisopliae y B. bassiana en el tratamiento T3.

De acuerdo con los resultados, aunque no se verificó la presencia de Paecilomyces sp a los 30 días, su aparición a los 60 días pudo haber estado relacionada con las condiciones de $\mathrm{pH}$ de 4.95 en el biol. Esto podría sugerir que existe compatibilidad de tres hongos en cada análisis, mientras que Paecilomyces sp., y Beauveria sp., no fueron observados juntos, por lo que se asume que exista una posible incompatibilidad. De acuerdo con Sharma et al. (2014), la mayor proliferación de Paecilomyces lilacinus fue alcanzada a valores de $\mathrm{pH}$ cercano a la neutralidad. Estudios previos han demostrado el efecto sobre la producción de metabolitos primarios y secundarios por parte de los microorganismos (Tamerler et al. 1998), los cuales podrían tener efecto de inducción o de antagonismo en el crecimiento de algunas poblaciones microbianas. Con base en estos estudios, se podría sugerir que posiblemente el valor de $\mathrm{pH}$ observado en el T2 pudo haber inducido algún mecanismo que favoreció el crecimiento de los hongos coexistiendo en el biol. 
La existencia de los microrganismos benéficos en niveles poblacionales altos a los 60 días después de la inoculación pudo favorecer la movilización del fósforo y el nitrógeno y la degradación de ácidos nucleicos y glicerofosfatos o fosfatos simples (Richardson \& Simpsom 2011). Díaz et al. (2009) deducen que algunos hongos y bacterias recuperan el nitrógeno, fósforo y otros micronutrientes que no pueden ser asimilados, lo vuelven disponibles para las plantas mejorando el intercambio catiónico. Estudios previos han demostrado que durante el proceso de fermentación, estos hongos liberan $\mathrm{CO}_{2}$ conjuntamente con el agua durante este proceso los fosfatos insolubles lo transforman en solubles.

En conclusión, los resultados evidenciaron que existe compatibilidad entre los hongos Trichoderma sp., Metarhizium sp., y Beauveria sp., mientras que este último no mostró compatibilidad aparente entre Paecilomyces sp., lo cual debería ser tomado en cuenta al momento de formular la incorporación de estos microorganismos en bioles artesanales. Por otra parte, el $\mathrm{pH}$ parece tener un rol fundamental en el crecimiento de las poblaciones de los microorganismos, por lo que se sugiere realizar estudios en los que se evalúe su efecto directo en el desarrollo poblacional de estos géneros de hongos y sus posibles efectos en la compatibilidad y/o antagonismo entre ellos.

\section{Conflictos de intereses}

Esta investigación se la realizó en la granja agroecológica del cantón Píllaro perteneciente al Honorable Consejo Provincial de Tungurahua Ecuador y no presenta conflictos de interés.

\section{Agradecimientos}

Los autores agradecen a la Universidad Técnica de Ambato. Facultad de Ciencias Agropecuarias y al Honorable Consejo Provincial de Tungurahua, por el apoyo técnico, científico y logístico realizado a la presente investigación.

\section{Literatura citada}

Arias EL, Piñeros PA. Aislamiento e identificación de hongos filamentosos de muestras del suelo de los páramos de Guasca y cruz verde. [Tesis de Licenciatura]. Pontificia Universidad Javeriana. Bogotá. 2008; 204 pp.

Bidlas E, Du T, Lambert RJW. An explanation for the effect of inoculum size on MIC and the Growth/No-Growth Interface. Int J Food Microbiol 2008; 126 (1-2): 140-152.

Campo-Martínez, ADP, Acosta-Sánchez RL, Morales-Velasco S. Evaluation of microorganisms of mountain $(\mathrm{mm})$ in the production of chard on the plateau of Popayán. Rev Bio Agro. 2014; 12(1); 79-87.

Díaz OA, Montero DM, Lagos JA. Acción de microorganismos eficientes sobre la actividad de intercambio catiónico en plántulas de acacia (Acacia melanoxylon) para la recuperación de un suelo del municipio de Mondoñedo, Cundinamarca. Colomb For 2009; 2 (1): 141-160.

Ferrera-Cerrato R, Alarcón A. La microbiología del suelo en la agricultura sostenible. Ciencia Ergo Sum 2001; 8(2): 175-183.

Leistner L. Basic aspects of food preservation by hurdle technology. Int J Food Microbiol 2000; 55, 181-186.

Navia CA, Cordova YZ, Morales S, Prado FA, López NA. Evaluation of different formula- 
tions from waste composting crop tomato (Solanum lycopersicum). Rev Bio Agro 2013; 11: 165-173.

Pino C. Determinación de la mejor dosis de Biol en el cultivo de (Musa sapientum) Banano, como alternativa a la fertilización química. [Tesis de Licenciatura]. Escuela Superior politécnica del Litoral. Guayaquil. 2009; 11 pp.

Richardson AE, Simpson RJ. Soil Microorganisms mediating phosphorus availability update on microbial phosphorus. Plant Physiol 2011; 156: 989-996.

Sánchez GL, Garzón MJ, Garzón MA, Giraldo FJ, Millán L, Villada ME. Aprovechamiento del suero lácteo de una empresa del norte antioqueño mediante microorganismos eficientes. $\mathrm{P}$ + L. 2009; 4 (2): 65-74.

Semu E, Singh BR. Accumulation of heavy metals in soils and plants after long-term use of fertilizers and fungicides in Tanzania. Fertil Res 1996; 44 (3): 241-248.
Sharma A., Sharma S., Mittal A., Naik S. Statistical optimization of growth media for Paecilomyces lilacinus 6029 using non-edible oil cakes. Ann Microbiol 2014; 64:515-520.

Tamerler C., Ullah M., Adlard M.W., Keshavarz T. Effect of $\mathrm{pH}$ on physiology of Metarhizium anisopliae for production of swainsonine. FEMS Microbiol Lett 1998; 168: 17-23.

Velastegui R. Alternativas ecológicas para el manejo integrado fitosanitario en los cultivos. Ed. Agro-Express Quito. 2005; 35-40.

Zapata JJ. Determinar la compatibilidad y el tiempo de sobrevivencia de tres microorganismos benéficos de uso agrícola: Beauveria bassiana, Bacilus thuringiensis y Paecilomyces lilacinus en compost. [Tesis de Maestría]. Universidad Técnica de Ambato. Querocha. 2015; 91 pp. 